\title{
Cramér-Rao-Type Bounds for Localization
}

\author{
Cheng Chang and Anant Sahai \\ Electrical Engineering and Computer Sciences, University of California, Berkeley, CA 94720, USA
}

Received 31 May 2005; Revised 10 November 2005; Accepted 1 December 2005

\begin{abstract}
The localization problem is fundamentally important for sensor networks. This paper, based on "Estimation bounds for localization" by the authors (2004 () IEEE), studies the Cramér-Rao lower bound (CRB) for two kinds of localization based on noisy range measurements. The first is anchored localization in which the estimated positions of at least 3 nodes are known in global coordinates. We show some basic invariances of the CRB in this case and derive lower and upper bounds on the CRB which can be computed using only local information. The second is anchor-free localization where no absolute positions are known. Although the Fisher information matrix is singular, a CRB-like bound exists on the total estimation variance. Finally, for both cases we discuss how the bounds scale to large networks under different models of wireless signal propagation.
\end{abstract}

Copyright ( 2006 Hindawi Publishing Corporation. All rights reserved.

\section{INTRODUCTION}

In wireless sensor networks, the positions of the sensors play a vital role. Position information can be exploited within the network stack at all levels from improved physical layer communication [1] to routing [2] and on to the application level where positions are needed to meaningfully interpret any physical measurements the sensors may take. Because it is so important, this problem of localization has been studied extensively. Most of these studies assume the existence of a group of "anchor nodes" that have a priori known positions. There are three major categories of localization schemes that differ in what kind of geometric information they use to estimate locations. Many, such as those of [3-7], use only the connectivity information reflecting whether node $i$ can directly communicate with node $j$ or anchor $k$. Such approaches are attractive because connectivity information is accessible at the network layer due to its use in multihop routing.

The second category uses both ranging and angular information for localization. Such schemes are studied in [810]. These are useful when there is a line of sight and antenna arrays are available at the sensor nodes so that beamforming is possible to determine the angles.

The third category is localization based solely on ranging measurements among nodes and between nodes and anchors. In $[11,12]$, the schemes for estimating ranges are discussed. References $[13,14]$ estimate the positions directly based on such node-to-anchor ranging estimates. In con- trast, $[15,16]$ first estimate positions in an anchor-free coordinate system and then embed it into the coordinate system defined by the anchors. In this paper we also focus on localization using ranging information alone.

The Cramér-Rao lower bound (CRB) [17] is widely used to evaluate the fundamental hardness of an estimation problem. The CRB for anchored localization using ranging information has been studied in [18-20]. The expression for the CRB was derived in [18]. In [20], a comparison of the CRB with the simpler Bayesian bound has been studied. In [19], simulation is used to study the impact of the density of the anchors and the size of the sensor network on the CRB.

As far as anchored localization goes, our additional contribution is giving a geometric interpretation of the CRB and deriving local lower and upper bounds on the CRB. The lower bounds imply that local geometry is critical for localization accuracy. The corresponding upper bounds show through simulation that the errors are not a lot worse if only the nearby anchors or nodes are involved in the position estimation of a particular node. These results show that distributed localization schemes are promising.

For anchor-free localization, as mentioned in [9], the Fisher information matrix (FIM) is singular and so the standard CRB analysis fails [21]. The CRB on anchor-free localization has not been thoroughly studied. In this paper, we give a geometric interpretation on a modified CRB and derive some properties of it. Furthermore, we show by example that anchor-free localization sometimes has a lower total estimation variance bound than anchored localization. 


\subsection{Outline of the paper}

After reviewing some basics in this introduction, Section 2 studies bounds for anchored localization. Assuming the ranging errors are i.i.d. Gaussian, we give an explicit expression for the FIM solely based on the geometry of the sensor network and show that the CRB is essentially invariant under zooming, translation, and rotation. Using matrix theory, we give a lower bound on the CRB that is determined by only local geometry. This converges to the $\mathrm{CRB}$ as the local area is expanded. We also give a corresponding local upper bound on the localization CRB. Finally we study the wireless situation in which the noise variance on the range measurements depends on the inter-sensor distance. Simulation results validate our intuition that the faster the signal decays, the less the CRB benefits from faraway information. A heuristic argument reveals the basic scaling laws involved.

Section 3 studies the bound for anchor-free localization. The rank of the FIM for $M$ nodes is shown to be at most $2 M-3$. The corresponding modified CRB is interpreted as a bound on the sum of the estimation variances. We observe that the per node bound in simulations appears to be proportional to the average number of neighbors and conjecture that the total estimation variance scales with the total received signal energy.

\subsection{Cramér-Rao bound on ranging}

Since range is our basic input, we first review the CRB for wireless ranging. The distance between two nodes is $c t_{d}$, where $c$ is the speed of light and $t_{d}$ is the time of arrival (TOA). TOA estimation is extensively studied in the radar literature. If $T$ is the observation duration, $A(t)$ is the pulse, ${ }^{1}$ and $N_{0}$ is the noise power spectral density, then for any unbiased estimate of $t_{d}[22]$,

$$
E\left[\left(\hat{t}_{d}-t_{d}\right)^{2}\right] \geq \frac{N_{0}}{\int_{0}^{T}[\partial A(t) / \partial t]^{2} d t} .
$$

Notice that $\int_{0}^{T}(\partial A(t) / \partial t)^{2} d t$ is proportional to the energy in the signal with the proportionality constant depending on the pulse shape. Because of the derivative, we know that having a pulse with a wide bandwidth is beneficial. Calling that proportionality $\tau_{r}^{2}$, we have

$$
E\left[\left(\hat{t}_{d}-t_{d}\right)^{2}\right] \geq \frac{\tau_{r}^{2}}{\mathrm{SNR}}
$$

The CRB on ranging is a fundamental bound coming only from the Gaussian thermal noise in the received signal. In reality, there are other sources of small ranging errors including interference, multipath spreading, unpredictable clock drifts,

\footnotetext{
${ }^{1}$ Notice that ranging estimates can be obtained from any pulse whose shape is known at the receiver. This includes data carrying packets that have been successfully decoded as long as we know the time they were supposed to have been transmitted. In a wireless sensor network, we are thus not restricted to use a dedicated radio for ranging.
}

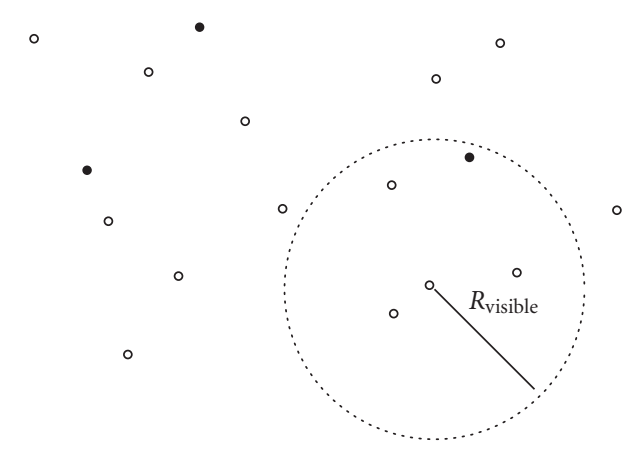

FIGURE 1: A sensor network. Solid dots are anchors; circles are nodes with unknown positions. The range $\hat{d}_{i, j}$ is estimated for sensor pairs $i, j$ s.t. $d_{i, j} \leq R_{\text {visible }}$.

operating system latencies, and so forth. These can cause the ranging error to be non-Gaussian even near the mean. More significantly, these ranging errors do not scale with SNR. We ignore all these other sources of error in this paper.

\subsection{Models of localization}

We idealize the localization problem by assuming all the sensors are fixed on a 2D plane. We have a set $S$ of $M$ sensors with unknown positions, together with a set $F$ of $N$ sensors (anchors) with known positions. Because the size of each sensor is assumed to be very small, it is treated as a point.

Each sensor generates limited-energy wireless signals that enable node $i$ to measure the distance to some nearby sensors in the set $\operatorname{adj}(i)$, as illustrated in Figure 1. We assume $j \in$ $\operatorname{adj}(i)$ if and only if $i \in \operatorname{adj}(j)$ for symmetry. Throughout, we also assume high $\mathrm{SNR}^{2}$ and so are free to assume that the distance measurements are only corrupted by independent zero mean Gaussian errors.

\subsubsection{Anchored localization}

If there are at least three nodes with positions known in global coordinates $(|F| \geq 3)$, then it is possible to estimate such global coordinates for each node using observations $D$ and position knowledge $P_{F}$ :

$$
\begin{gathered}
D=\left\{\widehat{d}_{i, j} \mid i \in S \cup F, j \in \operatorname{adj}(i)\right\}, \\
P_{F}=\left\{\left(x_{i}, y_{i}\right)^{T} \mid i \in F\right\} .
\end{gathered}
$$

Our goal is to estimate the set

$$
P_{S}=\left\{\left(\hat{x}_{i}, \hat{y}_{i}\right)^{T} \mid i \in S\right\}
$$

\footnotetext{
${ }^{2}$ Suppose that we are estimating the propagation time by looking for a peak in a matched filter. By high SNR we mean that the peak we find is in the near neighborhood of the true peak. At low SNR, it is possible to become confused due to false peaks arising entirely from the noise.
} 
$\left(x_{i}, y_{i}\right)$ is the position of sensor $i$. The measured distance between sensors $i$ and $j$ is $\hat{d}_{i, j}=\sqrt{\left(x_{i}-x_{j}\right)^{2}+\left(y_{i}-y_{j}\right)^{2}}+$ $\epsilon_{i, j}$, where $\epsilon_{i, j}$ 's are modeled as independent Gaussian errors $\sim N\left(0, \sigma_{i j}^{2}\right)$.

\subsubsection{Anchor-free localization}

If $|F|=0$, no nodes have known positions. This is an appropriate model whenever either we do not care about absolute positions, or if whatever global positions we do have are far more imprecise than the quality of measurements available within the sensor network. However, local coordinates are not unique. If $P_{S}=\left\{\left(\hat{x}_{i}, \hat{y}_{i}\right)^{T} \mid i \in S\right\}$ is a position estimate, then $P_{S}^{\prime}=\left\{R(\alpha)\left( \pm \hat{x}_{i}, \hat{y}_{i}\right)^{T}+(a, b)^{T} \mid i \in S\right\}$ is equivalent to $P_{S}$ where the \pm represents reflecting the entire network about the $y$ axis and $R(\alpha)$ is a rotation matrix:

$$
R(\alpha)=\left(\begin{array}{cc}
\cos (\alpha) & -\sin (\alpha) \\
\sin (\alpha) & \cos (\alpha)
\end{array}\right) .
$$

Thus, the performance measure for anchor-free localization should not be $\sum_{i}\left(x_{i}-\hat{x}_{i}\right)^{2}+\left(y_{i}-\hat{y}_{i}\right)^{2}$. The distance between equivalence classes should be used instead. Since the FIM for anchor-free localization is singular [9], the bound will be developed using the tools provided in [21].

\section{ESTIMATION BOUNDS FOR ANCHORED LOCALIZATION}

The Cramér-Rao bound (CRB) can be derived from the FIM.

\subsection{The anchored localization FIM}

In [18-20], expressions for the localization FIM were derived. The derivations are repeated below for completeness and furthermore, we observe that the FIM for localization is a function of the angles between nodes and anchors. As illustrated in Figure 2, the angle $\alpha_{i j} \in[0,2 \pi)$ from node $i$ to $j$ is defined as

$$
\begin{aligned}
& \cos \left(\alpha_{i j}\right)=\frac{x_{j}-x_{i}}{\sqrt{\left(x_{j}-x_{i}\right)^{2}+\left(y_{j}-y_{i}\right)^{2}}}=\frac{x_{j}-x_{i}}{d_{i j}}, \\
& \sin \left(\alpha_{i j}\right)=\frac{y_{j}-y_{i}}{\sqrt{\left(x_{j}-x_{i}\right)^{2}+\left(y_{j}-y_{i}\right)^{2}}}=\frac{y_{j}-y_{i}}{d_{i j}} .
\end{aligned}
$$

Let $x_{i}, y_{i}$ be the $(2 i-1)$ th and $2 i$ th parameters to be estimated, respectively, $i=1,2, \ldots, M$. The FIM is $J_{2 M \times 2 M}$.

Theorem 1 (FIM for anchored localization). For all $i=$ $1, \ldots, M$,

$$
\begin{gathered}
J_{2 i-1,2 i-1}=\sum_{j \in \operatorname{adj}(i)} \frac{\cos ^{2}\left(\alpha_{i j}\right)}{\sigma_{i j}^{2}}, \\
J_{2 i, 2 i}=\sum_{j \in \operatorname{adj}(i)} \frac{\sin ^{2}\left(\alpha_{i j}\right)}{\sigma_{i j}^{2}}, \\
J_{2 i-1,2 i}=J_{2 i, 2 i-1}=\sum_{j \in \operatorname{adj}(i)} \frac{\cos \left(\alpha_{i j}\right) \sin \left(\alpha_{i j}\right)}{\sigma_{i j}^{2}} .
\end{gathered}
$$

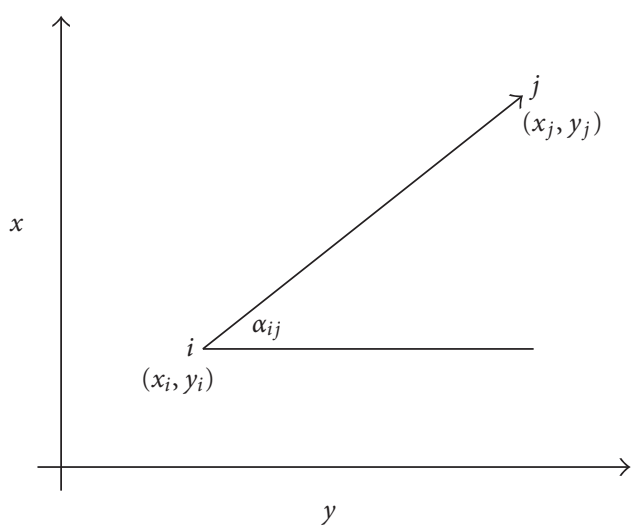

Figure 2: $\alpha_{i j}$ illustrated.

For nondiagonal entries $j \neq i$, if $j \in \operatorname{adj}(i)$,

$$
\begin{aligned}
J_{2 i-1,2 j-1} & =J_{2 j-1,2 i-1}=-\frac{1}{\sigma_{i j}^{2}} \cos ^{2}\left(\alpha_{i j}\right), \\
J_{2 i, 2 j} & =J_{2 j, 2 i}=-\frac{1}{\sigma_{i j}^{2}} \sin ^{2}\left(\alpha_{i j}\right), \\
J_{2 i-1,2 j} & =J_{2 j, 2 i-1}=J_{2 i, 2 j-1}=J_{2 j-1,2 i} \\
& =-\frac{1}{\sigma_{i j}^{2}} \sin \left(\alpha_{i j}\right) \cos \left(\alpha_{i j}\right)=-\frac{1}{2 \sigma_{i j}^{2}} \sin \left(2 \alpha_{i j}\right) .
\end{aligned}
$$

If $j \notin \operatorname{adj}(i)$, the entries are all zero.

Proof. We have the conditional pdf ${ }^{3}$

$$
p\left(\vec{d} \mid x_{1}^{M}, y_{1}^{M}\right)=\prod_{i<j, j \in \operatorname{adj}(i)} \frac{e^{-\left(\hat{d}_{i j}-d_{i j}\right)^{2} / 2 \sigma_{i j}^{2}}}{\sqrt{2 \pi \sigma_{i j}^{2}}} .
$$

The log likelihood is

$$
\ln \left(p\left(\vec{d} \mid x_{1}^{M}, y_{1}^{M}\right)\right)=C-\sum_{i<j, j \in \operatorname{adj}(i)} \frac{\left(\hat{d}_{i, j}-d_{i, j}\right)^{2}}{2 \sigma_{i j}^{2}}
$$

and so

$$
\begin{aligned}
J_{2 i-1,2 i-1} & =E\left(\frac{\partial^{2} \ln \left(p\left(\vec{d} \mid x_{1}^{M}, y_{1}^{M}\right)\right)}{\partial x_{i}^{2}}\right) \\
& =\sum_{j \in \operatorname{adj}(i)} \frac{1}{\sigma_{i j}^{2}}\left(\frac{x_{j}-x_{i}}{\sqrt{\left(x_{j}-x_{i}\right)^{2}+\left(y_{j}-y_{i}\right)^{2}}}\right)^{2} \\
& =\sum_{j \in \operatorname{adj}(i)} \frac{\cos ^{2}\left(\alpha_{i j}\right)}{\sigma_{i j}^{2}},
\end{aligned}
$$

and similarly for other entries of $J$.

\footnotetext{
${ }^{3} \vec{d}=\left\{\hat{d}_{i, j} \mid i<j, j \in \operatorname{adj}(i)\right\}$ is the observation vector. $x_{1}^{M}=\left(x_{1}, x_{2}, \ldots\right.$, $x_{M}$ ), similarly for $y_{1}^{M}$.
} 


\subsection{Properties of the anchored localization CRB}

Given the FIM, the CRB for any unbiased estimator is ${ }^{4}$

$$
\begin{aligned}
& E\left(\left(\hat{x}_{i}-x_{i}\right)^{2}\right) \geq J_{2 i-1,2 i-1}^{-1}, \\
& E\left(\left(\hat{y}_{i}-y_{i}\right)^{2}\right) \geq J_{2 i, 2 i}^{-1} .
\end{aligned}
$$

Corollary 1 (the FIM is invariant under zooming and translation $) . J\left(\left\{\left(x_{i}, y_{i}\right)\right\}\right)=J\left(\left\{\left(a x_{i}+c, a y_{i}+d\right)\right\}\right)$ for $a \neq 0$.

Proof. The angles $\alpha_{i j}$ and noise $\sigma_{i j}$ are unchanged and so the result follows immediately.

Corollary 2. The CRB for a single node is invariant under rotation and reflection: let $A=J\left(\left\{\left(x_{i}, y_{i}\right)\right\}\right), B=J\left(\left\{R\left(x_{i}, y_{i}\right)\right\}\right)$, where $R$ is a $2 \times 2$ matrix, with $R R^{T}=I_{2 \times 2}$. Then $A_{2 i-1,2 i-1}^{-1}+$ $A_{2 i, 2 i}^{-1}=B_{2 i-1,2 i-1}^{-1}+B_{2 i, 2 i}^{-1}$, for all $i=1,2 \ldots, M$.

Proof. Going through the derivation of the FIM, we find that $B=Q A Q^{T}$, where $Q$ is a $2 M \times 2 M$ matrix with the following form:

$$
\left(\begin{array}{cc}
Q_{2 i-1,2 i-1} & Q_{2 i-1,2 i} \\
Q_{2 i, 2 i-1} & Q_{2 i, 2 i}
\end{array}\right)=R
$$

with all other entries of $Q$ being 0 . Obviously $Q^{T} Q=Q Q^{T}=$ $I_{2 M \times 2 M}$ and so $B^{-1}=Q A^{-1} Q^{T}$. Write

$$
A(i)=\left(\begin{array}{cc}
A_{2 i-1,2 i-1}^{-1} & A_{2 i-1,2 i}^{-1} \\
A_{2 i, 2 i-1}^{-1} & A_{2 i, 2 i}^{-1}
\end{array}\right)
$$

and similarly for $B(i)$. Then $B(i)=R A(i) R^{T}$. Since $\operatorname{Tr}(X Y)=\operatorname{Tr}(Y X)$, we have $B_{2 i-1,2 i-1}^{-1}+B_{2 i, 2 i}^{-1}=\operatorname{Tr}(B(i))=$ $\operatorname{Tr}\left(R A(i) R^{T}\right)=\operatorname{Tr}\left(R^{T} R A(i)\right)=\operatorname{Tr}(A(i))=A_{2 i-1,2 i-1}^{-1}+$ $A_{2 i, 2 i}^{-1}$.

\subsection{A lower bound to the anchored localization CRB}

In order to invert the FIM and thereby evaluate the CRB, we need to take the geometry of the whole sensor network into account. In this section, we derive a performance bound for node $l$ that depends only on the local geometry around it. This has the potential to be valuable to "local" algorithms that try to do localization without performing all the computations in one center.

First we review a lemma for estimation variance.

Lemma 1 (submatrix bound). Let the row vector $\theta=\left(\theta_{1}\right.$, $\left.\theta_{2}, \ldots, \theta_{N}\right) \in R^{N}$; for all $M, 1 \leq M<N$, write $\theta^{*}=$ $\left(\theta_{N-M+1}, \ldots, \theta_{N}\right)$; then for any unbiased estimator for $\theta$,

$$
E\left(\left(\theta^{*}-\widehat{\theta^{*}}\right)^{T}\left(\theta^{*}-\widehat{\theta^{*}}\right)\right) \geq C^{-1},
$$

where $C$ is the $(N-M) \times(N-M)$ matrix:

$$
J(\theta)=\left(\begin{array}{cc}
A & B \\
B^{T} & C
\end{array}\right),
$$

\footnotetext{
${ }^{4}$ We write $\left(A^{-1}\right)_{i, j}$ as $A_{i, j}^{-1}$ for a nonsingular matrix $A$.
}

where $J(\theta)$ is the nonsingular, and hence positive definite, FIM for $\theta$.

Proof. Write the inverse of $J(\theta)$ as

$$
J(\theta)^{-1}=\left(\begin{array}{cc}
A^{\prime} & B^{\prime} \\
B^{\prime T} & C^{\prime}
\end{array}\right) .
$$

$J(\theta)$ is positive definite, then Theorem 5 in Appendix A guarantees

$$
C^{\prime} \geq C^{-1}
$$

The CRB theorem then gives $E\left(\left(\theta^{*}-\widehat{\theta^{*}}\right)^{T}\left(\theta^{*}-\widehat{\theta^{*}}\right)\right) \geq$ $C^{\prime} \geq C^{-1}$.

Notice that for any subset of $M$ nodes, we can always reorder them to get indices $N-M+1, \ldots, N$. By directly applying Lemma 1 we get the following.

Theorem 2 (a lower bound on the CRB). Write $\theta_{l}=\left(x_{l}, y_{l}\right)^{T}$ and write

$$
J_{l}=\frac{1}{\sigma^{2}}\left(\begin{array}{cc}
J(\theta)_{2 l-1,2 l-1} & J(\theta)_{2 l-1,2 l} \\
J(\theta)_{2 l, 2 l-1} & J(\theta)_{2 l, 2 l}
\end{array}\right) .
$$

Then for any unbiased estimator $\hat{\theta}, E\left(\left(\hat{\theta}_{l}-\theta_{l}\right)\left(\hat{\theta}_{l}-\theta_{l}\right)^{T}\right) \geq J_{l}^{-1}$.

This means we can give a bound on the estimation of $\left(x_{l}, y_{l}\right)$ using only the local geometry around sensor $l$.

Corollary 3. $J_{l}$ only depends on $\left(x_{l}, y_{l}\right)$ and $\left(x_{i}, y_{i}\right), i \in \operatorname{adj}(l)$.

Proof. $J_{l}$ in (7) only depends on $\left(\alpha_{l j}, \sigma_{l j}\right), j \in \operatorname{adj}(l)$. These only depend on $\left(x_{l}, y_{l}\right)$ and $\left(x_{i}, y_{i}\right)$.

Assume that the ranging errors are i.i.d. Gaussian with zero mean and common variance $\sigma^{2}$ and define the normalized FIM $K=\sigma^{2} J$. This is similar to the geometric dilution of precision (GDOP) in radar [23] since $K$ is dimensionless and only depends on the angles $\alpha_{i j}$ 's. Let $W=|\operatorname{adj}(l)|$ with sensors $\in \operatorname{adj}(l)$ being $l(1), \ldots, l(k), \ldots, l(W)$. Using elementary trigonometry and writing $\alpha_{k}=\alpha_{l, l(k)}$, we have

$$
J_{l}=\frac{1}{\sigma^{2}}\left(\begin{array}{cc}
\frac{W}{2}+\frac{\sum_{k=1}^{W} \cos \left(2 \alpha_{k}\right)}{2} & \frac{\sum_{k=1}^{W} \sin \left(2 \alpha_{k}\right)}{2} \\
\frac{\sum_{k=1}^{W} \sin \left(2 \alpha_{k}\right)}{2} & \frac{W}{2}-\frac{\sum_{k=1}^{W} \cos \left(2 \alpha_{k}\right)}{2}
\end{array}\right) .
$$

The sum of the estimation variance

$$
\begin{aligned}
E\left(\left(x_{l}-\hat{x}_{i}\right)^{2}+\left(y_{l}-\hat{y}_{i}\right)^{2}\right) \\
\quad \geq J_{l}^{-1}{ }_{11}+J_{l}^{-1}{ }_{22} \\
\quad=\frac{4 W \sigma^{2}}{W^{2}-\left(\sum_{k=1}^{W} \cos \left(2 \alpha_{k}\right)\right)^{2}-\left(\sum_{k=1}^{W} \sin \left(2 \alpha_{k}\right)\right)^{2}} \\
\quad \geq \frac{4 \sigma^{2}}{W}
\end{aligned}
$$




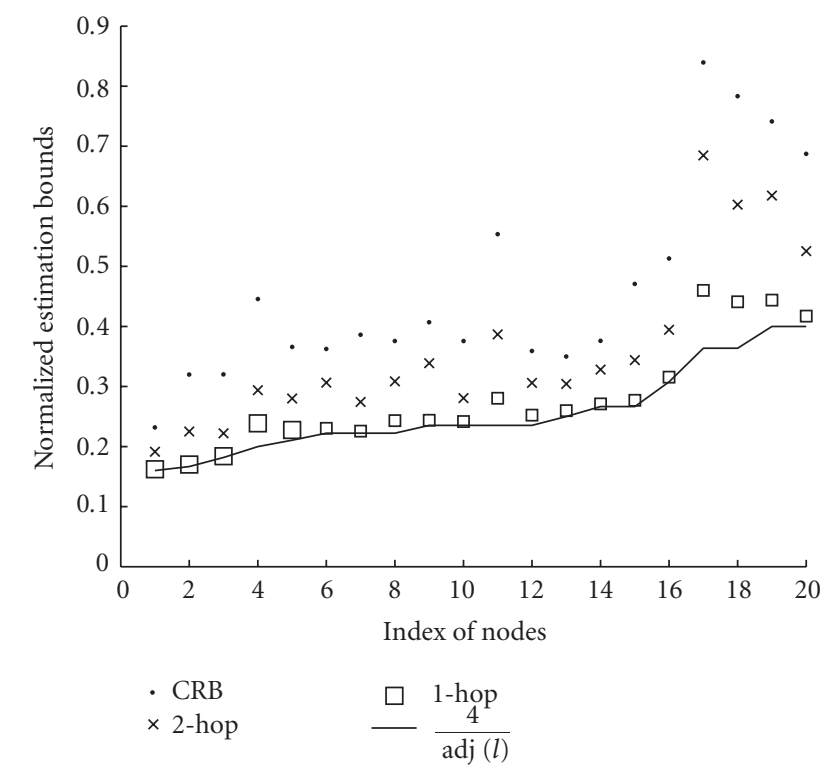

FIGURE 3: Bounds for 20 randomly chosen nodes indexed with decreasing $\operatorname{adj}(i)$.

with equality when $\sum_{k=1}^{W} \sin \left(2 \alpha_{k}\right)=0, \sum_{k=1}^{W} \cos \left(2 \alpha_{k}\right)=0$. This happens if the centroid of the unit vectors $\left(\cos \left(2 \alpha_{k}\right)\right.$, $\sin \left(2 \alpha_{k}\right)$ ) is the origin. A special case is when the angles $2 \alpha_{k}$ 's are uniformly distributed in $[0,2 \pi)$.

Above, we used one-hop geometric information around node $i$ to get a lower bound on the CRB. This bound can be interpreted as the CRB given perfect knowledge of the positions of all other nodes. ${ }^{5}$ We can use more information to tighten the bound. The lower bound using two-hop information is the CRB given the positions of all nodes $j, j \notin \operatorname{adj}(i)$, and similarly for multiple hops. The larger the local region we use to calculate the CRB is, the tighter it is. We define the CRB on such an estimation problem as the $N$-hop bound for that particular node. Obviously, the $N$-hop bound is nondecreasing with $N$, and the $\infty$-hop bound is the same as the CRB for the original estimation problem.

In our simulation, we have 200 nodes and 10 anchors all uniformly randomly distributed inside the unit circle, $j \in \operatorname{adj}(i)$, if and only if $d_{i \cdot j} \leq 0.3$. In Figure 3 , we plot the bounds for 20 randomly chosen nodes.

\subsection{An upper bound to the anchored localization CRB}

The CRB in Theorem 1 gives us the best performance an unbiased estimator can achieve given all information from the sensor network, including the positions of all anchors and all the available ranging information $\hat{d}_{i, j}$. This bounds the performance of a centralized localization algorithm where a central computer first collects all the information and then estimates the positions of the nodes.

\footnotetext{
${ }^{5}$ It is equivalent to knowing the positions of all the neighbors.
}

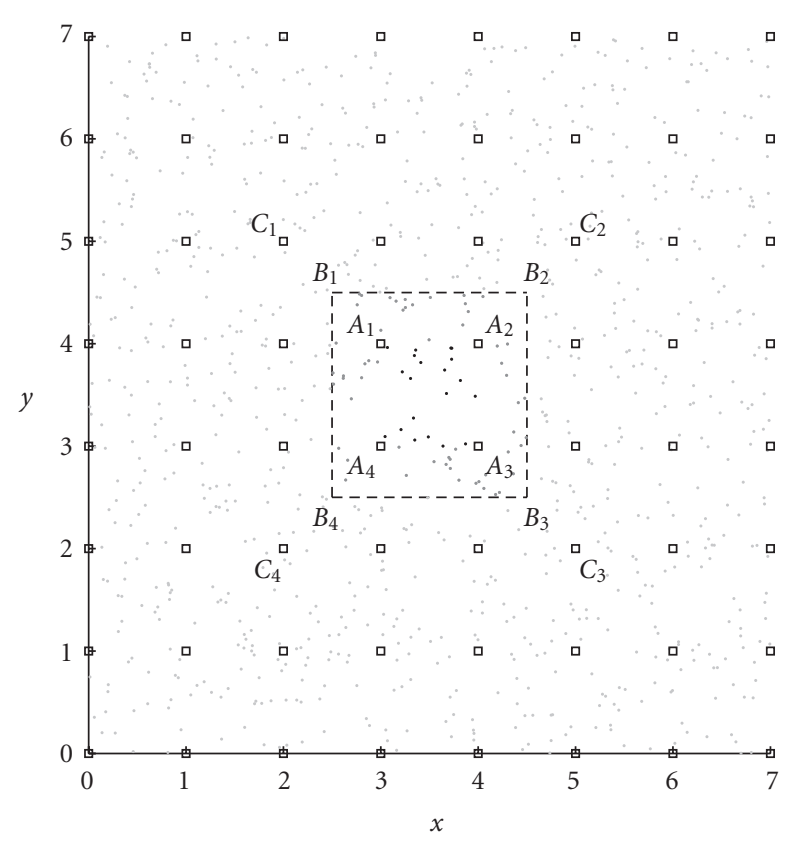

FIgURE 4: The setup of the sensor network anchors are shown as squares, nodes are shown as dots, nodes inside the central grid are shown as black dots.

In a sensor network, distributed localization is often preferred. In this "local" estimation problem only a subset of the anchors $F_{l} \subseteq F$ and a neighborhood of the nodes $l \in S_{l} \subseteq S$ may be taken into account. The CRB $V\left(x_{l}\right)$ and $V\left(y_{l}\right)$ of this local estimation problem computed from the $2\left|S_{l}\right| \times 2\left|S_{l}\right|$ FIM is an upper bound on the CRB for the original problem because strictly less information is used for estimation. ${ }^{6}$ In this section, the two bounds are compared through simulation.

The wireless sensor network is shown in Figure 4. Anchors are on the integer lattice points in a $7 \times 7$ square region. There are 20 nodes with unknown positions uniformly randomly distributed inside each grid square. Sensors $i$ and $j$ can see each other only if they are separated by a distance less than 0.5 .

We compute the normalized CRBs $\left(V_{i}=V_{i}^{x}+V_{i}^{y}\right.$, $i=1,2, \ldots, 20)$ for localization of the nodes inside the central grid $A_{1} A_{2} A_{3} A_{4}$ in 4 different cases corresponding to information from within the squares: $A_{1} A_{2} A_{3} A_{4}, B_{1} B_{2} B_{3} B_{4}$, $C_{1} C_{2} C_{3} C_{4}$, and the whole sensor network. As shown in Figure $5, V_{i}(A) \geq V_{i}(B) \geq V_{i}(C) \geq V_{i}(\mathrm{ALL}), i=1,2, \ldots, 20$. We observe that $V_{i}(C)$ (squares in Figure 5) is extremely close to $V_{i}$ (ALL) (the curve in Figure 5). More surprisingly, we observe that $V_{i}(B)$ is much smaller than $V_{i}(A)$.

To explore further, we gradually increase the size of the square region and compute the average CRB for $A_{1} A_{2} A_{3} A_{4}$.

\footnotetext{
${ }^{6}$ In [18], a rigorous proof is given for the equivalent proposition that the localization CRB for a node is nonincreasing as more nodes or anchors are introduced into the sensor network.
} 


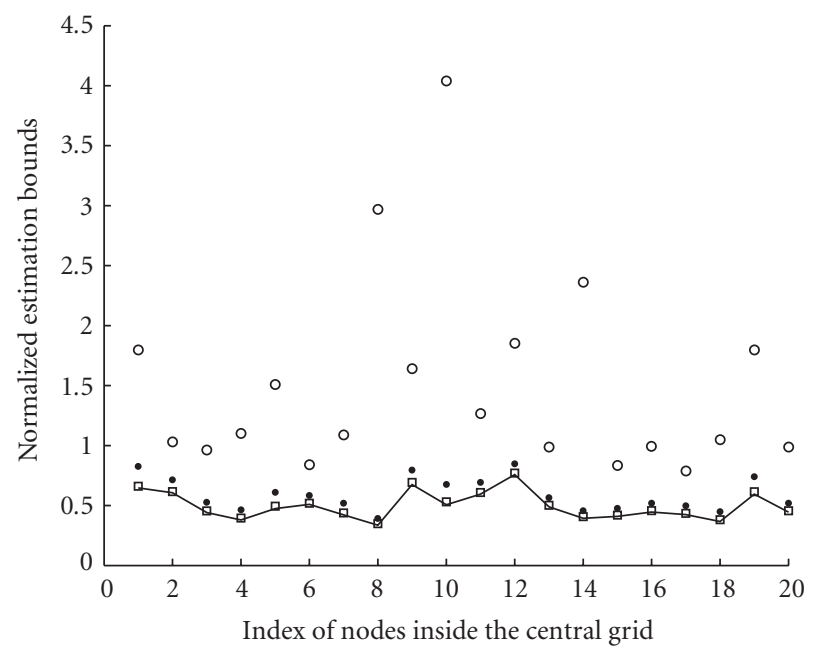

- Estimation bounds using the information inside $A_{1} A_{2} A_{3} A_{4}$

- Estimation bounds using the information inside $B_{1} B_{2} B_{3} B_{4}$

口 Estimation bounds using the information inside $C_{1} C_{2} C_{3} C_{4}$

_ Estimation bounds using all the information

Figure 5: Cramér-Rao bounds.

As shown in Figure 6, the average CRB decreases as the network size increases. After first dropping significantly, the upper bound levels off once we have included all the nodes directly adjacent to our neighborhood. This bodes well for doing distributed localization-distant anchors and ranging information do not significantly improve the estimation accuracy.

\subsection{CRB under different propagation models}

In the previous discussion, the ranging information was assumed to be corrupted by i.i.d. Gaussian errors. The ranging $\mathrm{CRB},(2)$, implies that the variance $\sigma_{i, j}^{2}$ of the additive noise on the distance measurement should depend on the distance $d_{i, j}$ between two nodes $i, j$, because the received wireless signal $A(t)$ attenuates as a function of $d$. We assume $\sigma_{i, j}^{2}=\sigma^{2} d_{i, j}^{a}$, where $\sigma^{2}$ is the noise variance when $d=1.7$ Furthermore, we assume a range estimate is available between all sensors, though it may be bad if they are far apart. Interference is ignored. This is reasonable only when there is no bandwidth constraint for the system as a whole, or if the data rates of communication are so low that all nodes can use signaling orthogonal to each other.

Define $K=\sigma^{2} J$ to be the normalized FIM. Just as in the case where $a=0$, translations of the whole sensor network do not change the FIM. Rotation does not change the CRB on any node $K_{2 i-1,2 i-1}^{-1}+K_{2 i, 2 i}^{-1}$. However, zooming does have an effect on the FIM.

Corollary 4 (the normalized FIM $K$ is scaled under zooming). If the propagation model is $d^{a}, a \geq 0$, and the

\footnotetext{
${ }^{7}$ Earlier, we had a hybrid model with $a=0$ locally and $a=\infty$ at a great distance since the range is only available for sensor pair $i, j$, if $d_{i, j}<R_{\text {visible. }}$.
}

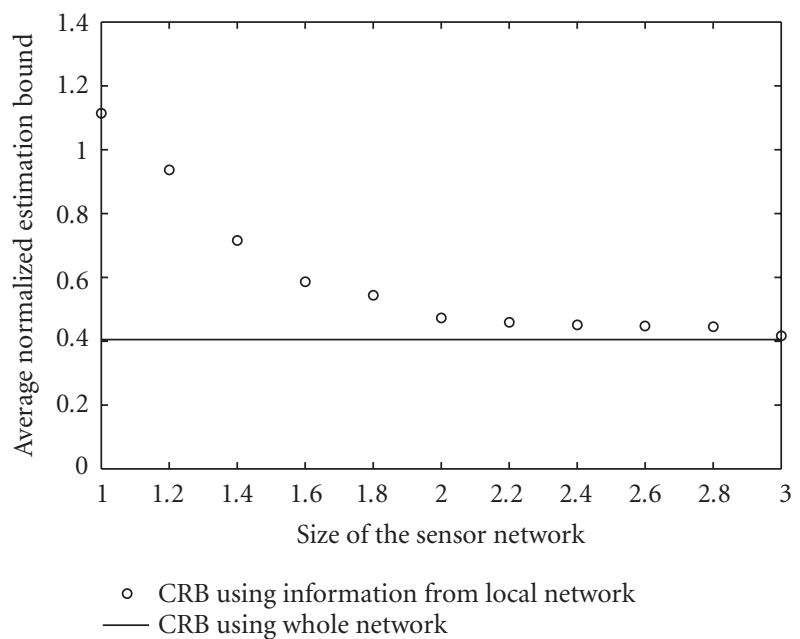

FiguRE 6: Average CRB versus sensor network size.

whole sensor network is zoomed by a zooming factor $c>0$, $K\left(\left\{c\left(x_{i}, y_{i}\right)\right\}\right)=\left(1 / c^{a}\right) K\left(\left\{\left(x_{i}, y_{i}\right)\right\}\right), c \neq 0$.

Proof. Zooming does not change the angles $\alpha_{i, j}$ between sensors. If the zooming factor is $c$, then the decaying factor changes to $\left(c d_{i, j}\right)^{a}=c^{a} d_{i, j}^{a}$. Substituting the new decaying factors into the FIM as in Theorem 1 , we get $K\left(\left\{c\left(x_{i}, y_{i}\right)\right\}\right)=$ $\left(1 / c^{a}\right) K\left(\left\{\left(x_{i}, y_{i}\right)\right\}\right)$.

The CRB $\sigma^{2} K_{i, i}^{-1}$ changes proportional to $c^{a}$, if the whole sensor network is zoomed up by a factor $c$.

Next, we have a simulation in which we fix the node density and examine the average CRB for different $a$ 's as we vary the size of the sensor network. The sensor network is the same as in Figure 4 and the sizes are taken at $1 \times 1,3 \times$ $3, \ldots, 13 \times 13$. We calculate the average CRB inside the central square and plot the average estimation bound in $10 \log _{10}$ scale in Figure 7.

The average CRB decreases as the size of the sensor network increases. This is expected since there is more information available and no interference by assumption. Asymptotically, the CRB decreases at a faster rate for smaller $a$ since the noise variance increases more slowly with range.

Heuristically, the localization accuracy for node $i$ is mainly determined by the total energy received by it. Suppose that the distance between nodes is $\geq r_{m}$, and the nodes are uniformly distributed. We approximate the total received energy $P_{R}$ coming from sensors within distance $R$ as

$$
\begin{aligned}
P_{R} & =\beta \int_{0}^{2 \pi} \int_{r_{m}}^{R} \rho^{-a} \rho d \rho d \theta=2 \beta \pi \int_{r_{m}}^{R} \rho^{1-a} d \rho \\
& = \begin{cases}\frac{2 \beta \pi}{2-a}\left(R^{2-a}-r_{m}^{2-a}\right) & \text { if } a \neq 2, \\
2 \beta \pi\left(\ln (R)-\ln \left(r_{m}\right)\right) & \text { if } a=2 .\end{cases}
\end{aligned}
$$

When $a<2, P_{R}$ behaves like $R^{2-a}$ which grows unboundedly as the network grows and similarly for $a=2$ where $P_{R}$ behaves like $\ln (R)$. In such nonphysical cases, it would be possible to save each node's transmitter power by going to a larger 


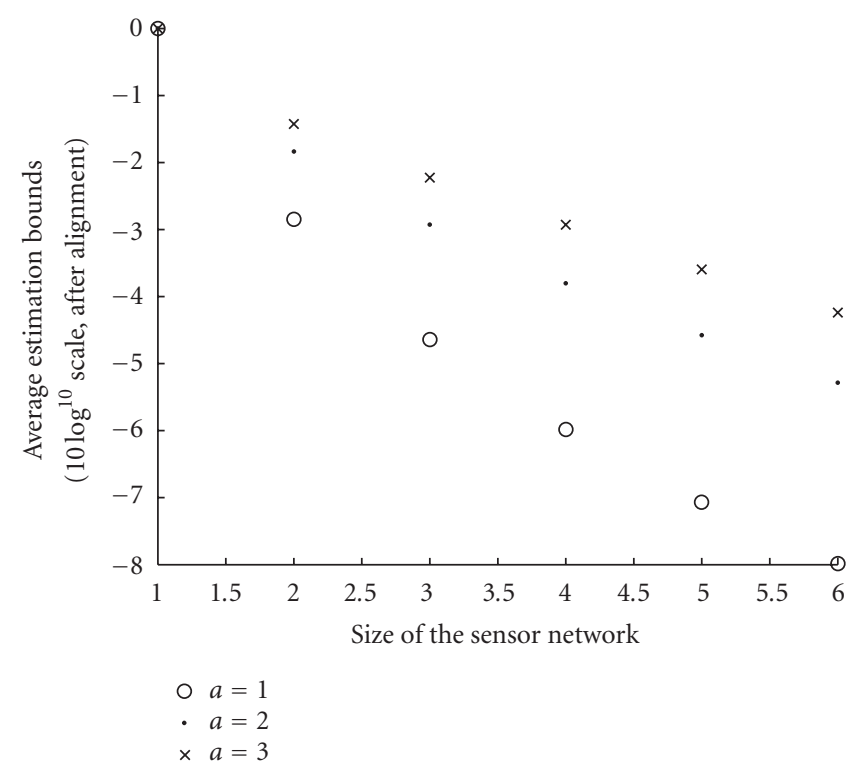

FIgUre 7: Average CRB in the central grid for different $a$. Circle: $a=1$, dot: $a=2$, cross: $a=3$.

network and then turning down the transmit power in such a way as to keep the position accuracy fixed. But in the physically relevant case of $a>2, P_{R}$ converges to $(2 \beta \pi /(a-2)) r_{m}^{2-a}$ and local measurements should be good enough. This heuristic explanation is a qualitative fit with simulations as illustrated in Figure 7.

\section{ESTIMATION BOUNDS FOR ANCHOR-FREE LOCALIZATION}

For anchor-free localization, only the inter-node distance measurements are available. The nature of anchor-free localization is very different from anchored localization, in that the absolute positions of the nodes cannot be determined. We first review the singularity of the FIM using the treatment from [17].

Lemma 2 (rank of the FIM). Let $\vec{d}$ be the observation vector, and let $\theta$ be the $n$-dimensional parameter to be estimated. Write the log likelihood function as $l(\vec{d} \mid \theta)=\ln (p(\vec{d} \mid \theta))$. The rank of the FIM $J$ is $n-k, k \geq 0$, if and only if the expectation of the square of directional derivative of $l(\vec{d} \mid \theta)$ at $\theta$ is zero for $k$ independent vectors $b_{1}, \ldots, b_{k} \in R^{n}$.

Proof. The directional derivative of $l(\vec{d} \mid \theta)$ at $\theta$, along direction $b_{i}$ is $\tau\left(b_{i}\right)=\left(\partial l / \partial \theta_{1}, \partial l / \partial \theta_{2}, \ldots, \partial l / \partial \theta_{n}\right) b_{i}$.

$$
\begin{aligned}
& E\left(\tau\left(b_{i}\right)^{2}\right) \\
& \quad=E\left(b_{i}^{T}\left(\partial l / \partial \theta_{1}, \ldots, \partial l / \partial \theta_{n}\right)^{T}\left(\partial l / \partial \theta_{1}, \ldots, \partial l / \partial \theta_{n}\right) b_{i}\right) \\
& \quad=b_{i}^{T} J b_{i} .
\end{aligned}
$$

If $k$ independent vectors $b_{1}, \ldots, b_{k}$ make $b_{i}^{T} J b_{i}=0$, the rank of $J$ is $n-k$, since $J$ is an $n \times n$ symmetric matrix.

The FIM for anchor-free localization is given in Theorem 1 , just with no anchors. With the above lemma, we can prove that the rank of this FIM is deficient by at least 3. This is intuitively clear since there are 3 degrees of freedom coming from rotation and translation.

Theorem 3. For the anchor-free localization problem, with $M$ nodes, the FIM $J(\theta)$ is of rank $2 M-3$.

Proof. The log-likelihood function of this estimation problem is

$$
\begin{aligned}
& l(\vec{d} \mid \theta) \\
& =\ln \left(p \left(\left\{\hat{d}_{i, j}, 1 \leq i, j \leq M, j \in \operatorname{adj}(i)\right\} \mid\right.\right. \\
& \left.\left.\quad\left\{\sqrt{\left(x_{i}-x_{j}\right)^{2}+\left(y_{i}-y_{j}\right)^{2}}, 1 \leq i, j \leq M, j \in \operatorname{adj}(i)\right\}\right)\right) \\
& =\sum_{1 \leq i, j \leq M, j \in \operatorname{adj}(i)} \ln \left(p\left(\hat{d}_{i, j} \mid \sqrt{\left(x_{i}-x_{j}\right)^{2}+\left(y_{i}-y_{j}\right)^{2}}\right)\right) .
\end{aligned}
$$

The last equality comes from the independence of the measurement errors. The directional derivative of each term in the sum is 0 along the vectors $\vec{b}_{1}, \vec{b}_{2}, \vec{b}_{3} \in R^{2 M}$. $\vec{b}_{1}=(1,0,1,0, \ldots, 1,0)^{T}, \vec{b}_{2}=(0,1,0,1, \ldots, 0,1)^{T}, \vec{b}_{3}=$ $\left(y_{1},-x_{1}, y_{2},-x_{2}, \ldots, y_{M},-x_{M}\right)^{T}$ where $\vec{b}_{1}$ and $\vec{b}_{2}$ span the $2 \mathrm{D}$ space in $R^{2 M}$ corresponding to translations and $\vec{b}_{3}$ is the instantaneous direction when the whole sensor networks rotates.

Since the FIM is not full rank, we cannot apply the standard CRB argument because $J^{-1}$ does not exist. Instead, the $\mathrm{CRB}$ is the Moore-Penrose pseudo-inverse $J^{\dagger}[21]$.

\subsection{The meaning of $\mathrm{J}^{\dagger}$ : the total estimation bound}

When the FIM is singular, we cannot properly define the parameter estimation problem in $R^{n}$. However, we can estimate the parameters in the local subspace spanned by all $k$ orthonormal eigenvectors $\vec{v}_{1}, \ldots, \vec{v}_{k}$ corresponding nonzero eigenvalues of $J$. In that subspace, the FIM $Q$ is full rank. Write $V=\left(v_{1}, \ldots, v_{k}\right), V$ is an $n \times k$ matrix and $V^{T} V=I_{k}$; then $Q=V^{T} J V$, and $Q^{-1}=V^{T} J^{\dagger} V$; thus $J^{\dagger}$ is the intrinsic $\mathrm{CRB}$ matrix for the estimation problem. The total estimation bound for the estimation problem in the $k$-dimensional subspace is $\operatorname{Tr}\left(Q^{-1}\right)$, and $\operatorname{Tr}\left(Q^{-1}\right)=\operatorname{Tr}\left(J^{\dagger}\right)$ by elementary matrix theory.

Unlike the anchored case, we cannot claim the estimation accuracy of a single node to be bounded by

$$
E\left(\left(\hat{x}_{i}-x_{i}\right)^{2}\right)+E\left(\left(\hat{y}_{i}-y_{i}\right)^{2}\right) \geq J_{2 i-1,2 i-1}^{\dagger}+J_{2 i, 2 i}^{\dagger}
$$

since there always exists a translation of the entire network 
to make the estimation of node $i$ perfectly accurate. However, the total estimation bound constrains the performance of anchor-free localization since the trace is invariant. ${ }^{8}$

Definition 1. Total estimation bound $V_{\text {total }}(J)$ on anchor-free localization $^{9}$ is as follows:

$$
V_{\text {total }}(J)=\sum_{i=1}^{M}\left(J_{2 i-1,2 i-1}^{\dagger}+J_{2 i, 2 i}^{\dagger}\right)=\operatorname{Tr}\left(J^{\dagger}\right)
$$

By the definition we know that $V_{\text {total }}(K)$ is invariant under rotation, translation, and zooming.

Theorem 4 (total estimation bound $V_{\text {total }}(J)$ on an anchor-free localization problem). $V_{\text {total }}(J)=\sum_{i=1}^{2 M-3}\left(1 / \lambda_{i}\right)$, where $\lambda_{i}$ 's are nonzero eigenvalues of $J$.

Proof. The correctness follows the fact that the eigenvalues of $J^{\dagger}$ are $1 / \lambda_{1}, 1 / \lambda_{2}, \ldots, 1 / \lambda_{2 M-3}, 0,0,0$. And so $\operatorname{Tr}\left(J^{\dagger}\right)=$ $\sum_{i=1}^{2 M-3}\left(1 / \lambda_{i}\right)$.

\subsubsection{Total estimation bound on 3-node anchor-free localization}

Using Theorem 4, we can give the total lower bound on any geometric setup of an anchor-free localization. The simplest nontrivial case is when there are only 3 points. We fix two points at $(0,0),(0,1)$. We plot the contour of the total estimation bound as a function of the position of the $3 \mathrm{rd}$ node $\in[0,1] \times[0,1]$.

The result shows that the total estimation bound is related to the biggest angle of the triangle. The larger that angle is, the larger the total estimation bound is. From Figure 8, we find that the minimum total estimation bound is achieved when the triangle is equilateral, where the 3 rd node is at $(0.5, \sqrt{3} / 2)$. Figure 9 (b) shows what is happening around the minimum.

\subsubsection{Total estimation bound for different network shapes}

The shape of the sensor network affects the total estimation bound. We illustrate this by a simulation with $M$ sensors randomly and uniformly distributed in a region with all the pairwise distances measured. We plot the average normalized total estimation bound of 50 independent experiments.

Figure 10 reflects a rectangular region with dimension $L_{1} \times L_{2}, L_{1} \geq L_{2}$. Since the zooming does not change the total estimation bound, only the ratio $R=L_{1} / L_{2}$ matters and

\footnotetext{
${ }^{8}$ A geometric interpretation of this total estimation is as follows. Imagine that the estimation is done in the $(2 n-3)$-dimensional subspace which is orthogonal to the 3 -dimensional space spanned by $\vec{b}_{1}, \vec{b}_{2}, \vec{b}_{3}$. Then the expectation of the square of the error vector will be upper bounded by $\operatorname{Tr}\left(J^{\dagger}\right)$.

${ }^{9}$ For anchored localization, $J$ is nonsingular. Thus $J^{-1}=J^{\dagger}$. It is immediate from the definition of the CRB that $\sum_{i} E\left(\left(\hat{x}_{i}-x_{i}\right)^{2}+\left(\hat{y}_{i}-y_{i}\right)^{2}\right) \leq \operatorname{Tr}\left(J^{-1}\right)=$ $\operatorname{Tr}\left(J^{\dagger}\right)$.
}

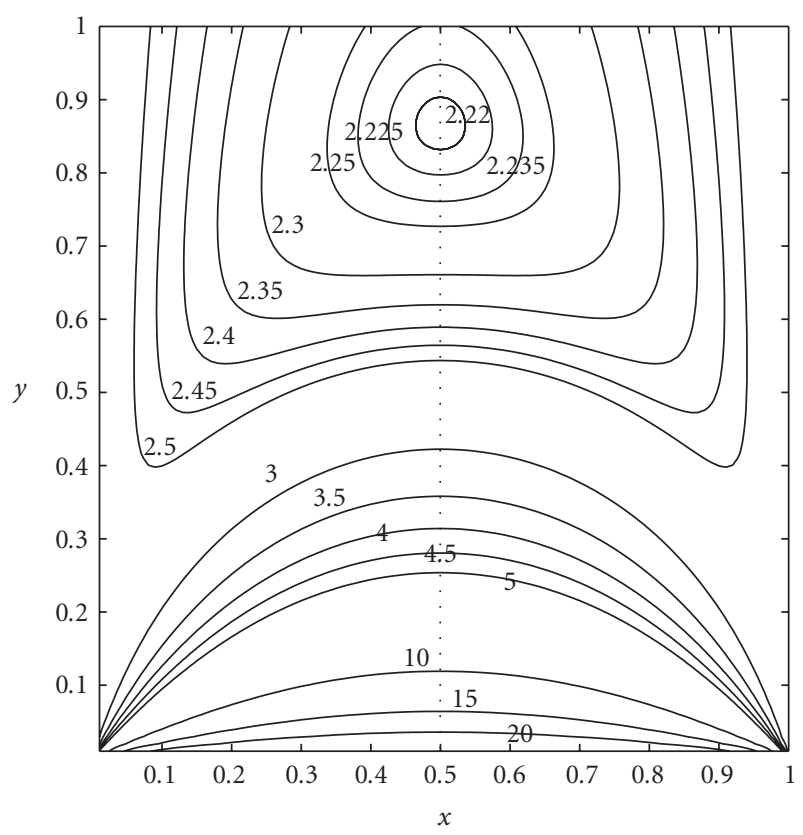

FIgURE 8: The contour shows the total estimation bound in $10 \log _{10}$ scale for the 3rd node at $(x, y)$.

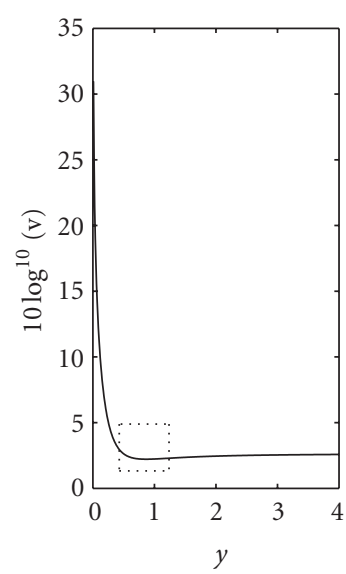

(a)

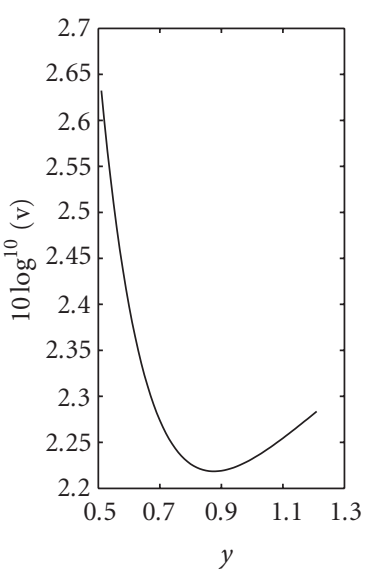

(b)
FIgure 9: The total estimation bound. The 3 rd node is at $(0.5, y)$ along the dotted line in Figure 8.

it turns out that the normalized CRB increases as $R$ increases, or as the rectangle becomes less and less square. ${ }^{10}$ However, once the number of nodes had gotten large enough, the total estimation error bound did not change with more nodes. The error was reduced per-node in a way that simply distributed the same total error over a larger number of nodes.

\footnotetext{
${ }^{10}$ In [24], we also studied the total estimation bound for an annular region. Let $R=r_{\text {inner }} / r_{\text {outer }}$ be the ratio of the radius of the inner circle over the radius of the outer circle, we observe that the total estimation bound decreases as $R$ increases and again the total estimation bound is roughly constant with respect to the number of nodes. The best case is having the nodes along the circumference of a circle!
} 


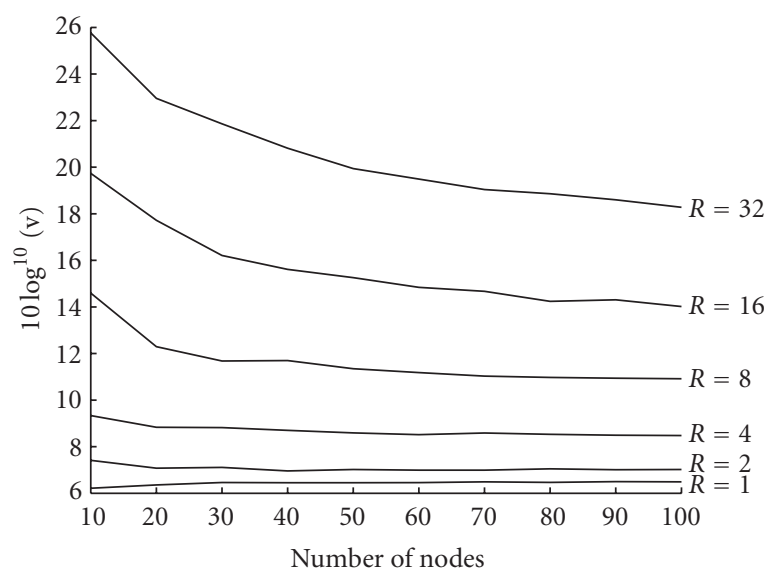

Figure 10: The normalized total estimation lower bound versus number of nodes. Rectangular region $\left(R=L_{1} / L_{2}\right)$.

\subsection{Why not set a node at $(0,0)$ and another node on the $x$ axis}

It is tempting to eliminate the singularity of the FIM by just setting some parameters. If we fix node 1 at position $\left(x_{1}, y_{1}\right)$, node 2 with $y$-coordinate 0 , it is equivalent to doing the estimation in the subspace through point $\left(x_{1}, y_{1}, \ldots, x_{M}, y_{M}\right)$ perpendicular to $\vec{c}_{1}=(1,0,0,0, \ldots, 0)^{T}$, $c_{2}=(0,1,0,0, \ldots, 0)^{T}, \vec{c}_{3}=(0,0,1,0, \ldots, 0)^{T}$. In general, the subspace generated by $\overrightarrow{c_{1}}, \overrightarrow{c_{2}}, \overrightarrow{c_{3}}$ is not the same as that generated by $\vec{b}_{1}, \vec{b}_{2}, \vec{b}_{3}$ and so the choice of which nodes we choose to fix can impact the bounds!

\subsection{Comparison of anchored and anchor-free localization}

Sometimes a bad geometric setup of anchors results in bad anchored estimation, while the anchor-free estimation is still good! As such, it is not useful to view the anchor-free case as an information-limited version of the anchored case. After all, in the anchored case, we also have a more challenging goal: to get the absolute positions correct, not just up to equivalency. In Figure 11, we have a sensor network with 3 anchors very close to each other; the total estimation bound for anchored localization is 195.20; meanwhile the total estimation bound for anchor-free localization is $4.26 .{ }^{11}$

\subsection{Total estimation bound under different propagation models}

It can be easily seen that just as in the anchored localization, $J$ is invariant under translation and $V_{\text {total }}(J)$ is invariant under rotation as well. Just as in anchored localization, the total estimation bound $V_{\text {total }}(J)$ changes proportional to $c^{a}$, if the whole sensor network is zoomed up by a factor $c$.

${ }^{11}$ As a result, we suggest that algorithm designers avoid fixing the global coordinate system unless they are confident on the setup of the anchors.

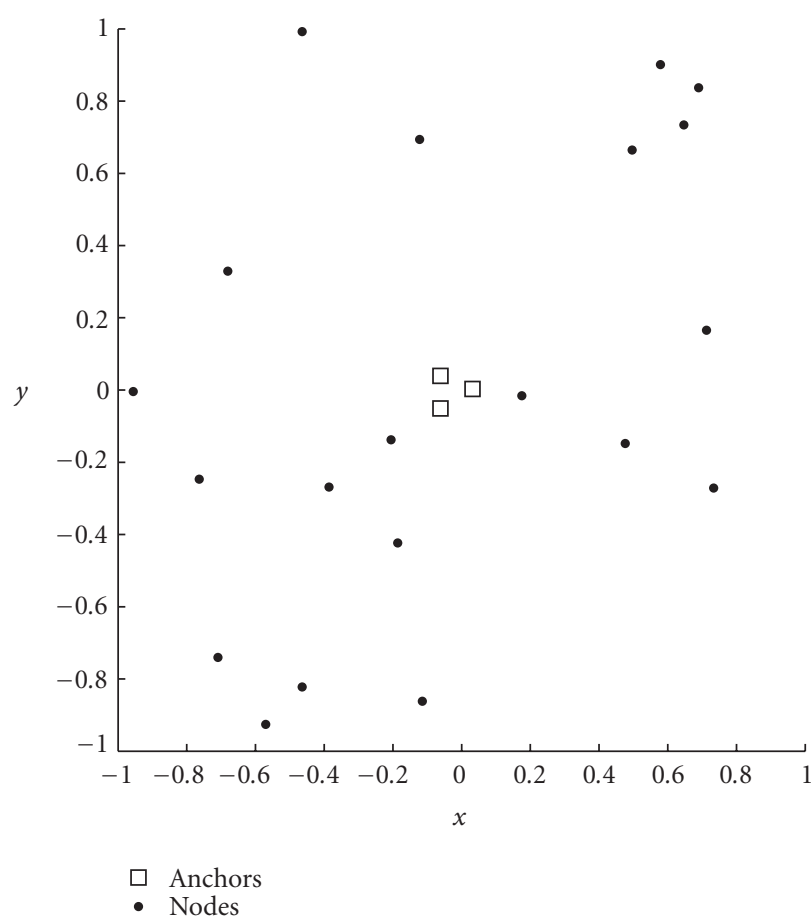

FIgURE 11: A bad setup of anchors.

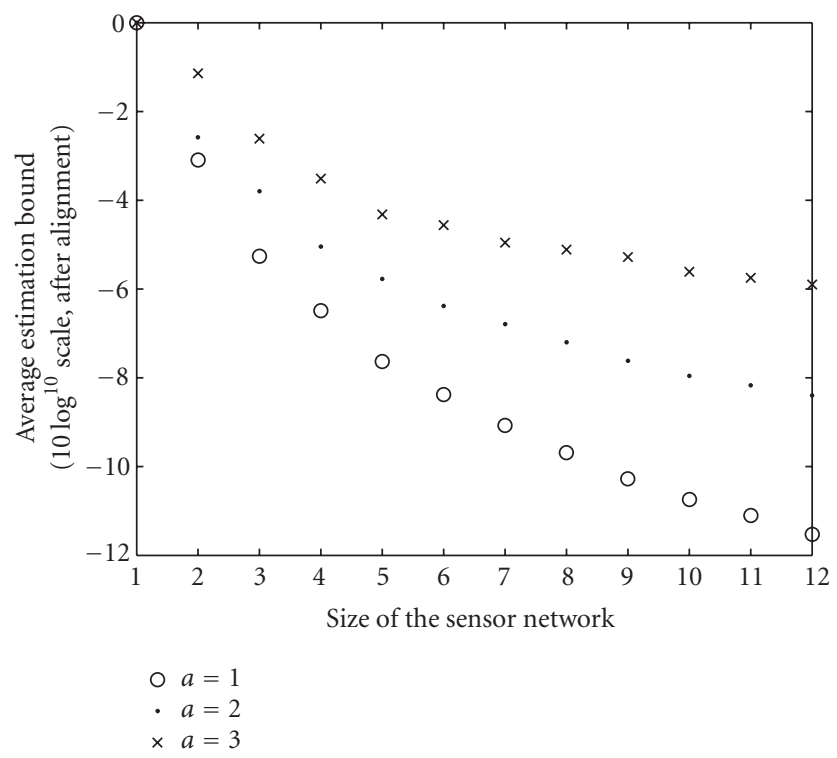

FIGURE 12: The average normalized total estimation lower bound versus size of the sensor network for different $a$.

In simulation, we study the effect of the size of the sensor network on the average estimation bound in different propagation models, that is, for different $a$ 's using the same setup as Figure 4.

As shown in Figure 12, we observe that the average estimation bound decreases as the size of the sensor network increases with fixed node density. Just as in the anchored case shown in Figure 7, the estimation accuracy is mainly 
determined by the received power and so the heuristic explanation for the anchored case also fits the simulation results we have for the anchor-free case.

\section{CONCLUSIONS AND FUTURE WORK}

In this paper, we studied the CRB for both anchored and anchor-free localization and gave a method to compute the CRB in terms of the geometry of the sensor network. For anchored localization, we derived both lower and upper bounds on the CRB which are determined by only local geometry. These showed that we can use local geometry to predict the accuracy of the position estimation that bodes well for distributed algorithms. The implication of our results on sensor network design is that accurate position estimation requires good local geometry of the sensor network. For anchor-free localization, the singularity of the FIM was overcome by computing the total estimation bound instead. Finally, we considered the implications of wireless signal propagations and found that if the signals propagate very well, then there are potentially significant gains by using larger networks and doing estimation in a manner that uses this information. However, such path-loss models are unphysical and so practical schemes should work fine with only local information.

So far, we have only computed the CRB. For the design of algorithms, it would also be good to know the sensitivity of the bound to individual observations. It might be very helpful in localization if one can identify the bottlenecks of the problem, that is, figure out which distance measurement could help to increase the localization accuracy the most. With the knowledge of the bottlenecks, it may be possible to allocate the energy or computation in a smart way to improve localization accuracy. Finally we do not know if we can approach the bound with distributed or centralized localization. ${ }^{12}$

\section{APPENDICES}

\section{A. PROOF OF $(20)$}

The lemmas and the theorem in the appendix can be treated as corollaries of the results in [25]. We prove all the lemmas and the theorem here for self completeness.

Theorem 5. For a positive definite $N \times N$ matrix $J$,

$$
J=\left(\begin{array}{cc}
A & B \\
B^{T} & C
\end{array}\right)
$$

where $A$ is an $M \times M$ symmetric matrix, $C$ is an $(N-M) \times$ $(N-M)$ symmetric matrix, and $B$ is an $M \times(N-M)$ matrix, if we write

$$
J^{-1}=\left(\begin{array}{cc}
A^{\prime} & B^{\prime} \\
B^{\prime T} & C^{\prime}
\end{array}\right),
$$

\footnotetext{
12 In Appendix B, we compare the CRB of anchored localization with the biased-localization scheme proposed in [24] to illustrate another important caveat.
}

where $A$ and $A^{\prime}$ have the same size, $B$ and $B^{\prime}$ have the same size, so do $C$ and $C^{\prime}$. $C^{\prime}-C^{-1}$ is positive semidefinite.

First we need several lemmas.

Lemma 3. $A$ is positive definite.

Proof. For all $\vec{x} \in R^{M}, x \neq 0$. Let $\vec{y}=(\vec{x}, \overrightarrow{0})^{T}$, where $\overrightarrow{0}$ is the $1 \times(N-M)$ all 0 vector, $\vec{y}$ is an $N$-dimensional vector. Then $\vec{x}^{T} A \vec{x}=\vec{y}^{T} J \vec{y}>0$.

The last inequality is true because $J$ is positive definite, and $\vec{y} \neq 0 . \vec{x}$ is arbitrary, so $A$ is positive definite.

Similarly $C$ is positive definite, and thus $A, C$ are nonsingular.

Lemma 4. $A-B C^{-1} B^{T}$ is positive definite.

Proof. First notice that for a positive definite matrix $J$, $J$ can be written as $J_{H}^{T} J_{H}$, where $J_{H}$ is an $N \times N$ nonsingular matrix. Write $J_{H}=\left(\begin{array}{ll}S & R\end{array}\right)$, where $S$ is an $N \times M$ matrix and $R$ is an $N \times(N-M)$ matrix. Then

$$
A=S^{T} S ; \quad B=S^{T} R ; \quad C=R^{T} R ;
$$

$C$ is nonsingular, so $R$ has full rank $N-M$. The singular value decomposition of $R$ is $R=U \Lambda V$, where $U$ is an $N \times N$ matrix, $U^{T} U=U U^{T}=I, V$ is an $(N-M) \times(N-M)$ matrix, $V^{T} V=V V^{T}=I$, and $\Lambda$ is an $N \times(N-M)$ matrix.

$$
\Lambda=\left(\begin{array}{c}
\operatorname{diag}\left(\lambda_{1}, \ldots, \lambda_{N-M}\right) \\
0_{M \times(N-M)}
\end{array}\right)
$$

$\lambda_{i}>0$ because $R$ has full $\operatorname{rank} N-M$. Now

$$
\begin{aligned}
A-B & C^{-1} B^{T} \\
& =S^{T} S-S^{T} R\left(R^{T} R\right)^{-1} R^{T} S \\
& =S^{T}\left(I-R\left(R^{T} R\right)^{-1} R^{T}\right) S \\
& =S^{T}\left(I-(U \Lambda V)\left((U \Lambda V)^{T}(U \Lambda V)\right)^{-1}(U \Lambda V)^{T}\right) S \\
& =S^{T}\left(I-U \Lambda V\left(V^{T} \Lambda^{T} \Lambda V\right)^{-1} V^{T} \Lambda^{T} U^{T}\right) S \\
& =S^{T}\left(I-U \Lambda V V^{T}\left(\Lambda^{T} \Lambda\right)^{-1} V^{T} V \Lambda^{T} U^{T}\right) S \\
& =S^{T}\left(I-U \Lambda\left(\Lambda^{T} \Lambda\right)^{-1} \Lambda^{T} U^{T}\right) S \\
& =S^{T} U\left(I-\Lambda\left(\Lambda^{T} \Lambda\right)^{-1} \Lambda^{T}\right) U^{T} S=S^{T} U \Delta U^{T} S,
\end{aligned}
$$

where $\Delta=\operatorname{diag}\left(\delta_{1}, \delta_{2}, \ldots, \delta_{N}\right)$, where $\delta_{i}=0, i=$ $1,2, \ldots, N-M$ and $\delta_{i}=1, N-M<i \leq N$. Obviously $A-B C^{-1} B^{T}$ is positive semidefinite. Suppose $\exists \vec{x} \in R^{M}$, $\vec{x} \neq 0$, but $\vec{x}^{T} S^{T} U \Delta U^{T} S \vec{x}=0$. Then we have $U^{T} S \vec{x}=$ $\left(y_{1}, y_{2}, \ldots, y_{N}\right)^{T}=\vec{y}$ and $y_{N-M+1}, \ldots, y_{N}$ all equal to 0 . Now $S \vec{x}=U \vec{y}$, and from the fact that $y_{N-M+1}, \ldots, y_{N}$ all equal to 0 , we have $\Lambda\left(\Lambda^{T} \Lambda\right)^{-1} \Lambda^{T} \vec{y}=\vec{y}$. Write $\vec{z}=V^{T}\left(\Lambda^{T} \Lambda\right)^{-1} \Lambda^{T} \vec{y}$, then $S \vec{x}=U \vec{y}=U \Lambda V \vec{z}=R \vec{z}$, where $\vec{x} \neq 0$. This contradicts the fact that $(S R)$ is full rank.

Similarly, $C-B^{T} A^{-1} B$ is positive definite, and thus both $C-B^{T} A^{-1} B$ and $A-B C^{-1} B^{T}$ are full rank. 


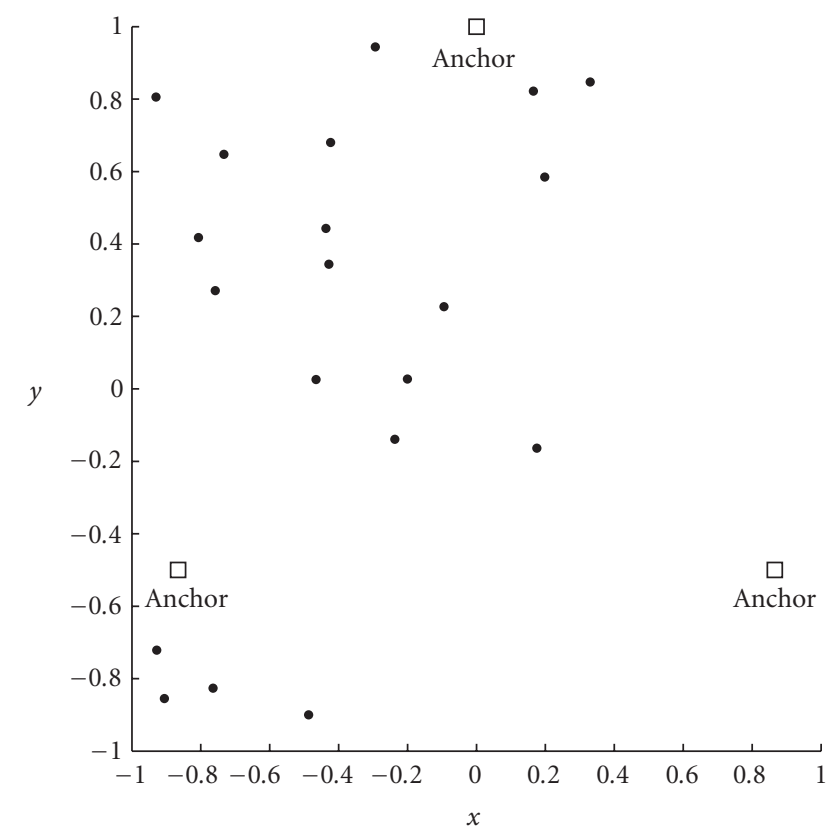

FIGURE 13: Setup of the sensor network $M=20$.

Lemma 5. $\left(C-B^{T} A^{-1} B\right)^{-1}=C^{-1} B^{T}\left(A-B C^{-1} B^{T}\right)^{-1} B C^{-1}+$ $C^{-1}$.

Proof. Notice that both $A$ and $\left(A-B C^{-1} B^{T}\right)$ are full rank; then,

$$
\begin{gathered}
\left(C^{-1} B^{T}\left(A-B C^{-1} B^{T}\right)^{-1} B C^{-1}+C^{-1}\right)\left(C-B^{T} A^{-1} B\right) \\
=I+C^{-1} B^{T}\left(A-B C^{-1} B^{T}\right)^{-1} B-C^{-1} B^{T} A^{-1} B \\
\\
-C^{-1} B^{T}\left(A-B C^{-1} B^{T}\right)^{-1} B C^{-1} B^{T} A^{-1} B \\
=I+C^{-1} B^{T}\left(\left(A-B C^{-1} B^{T}\right)^{-1}-A^{-1}\right. \\
\left.-\left(A-B C^{-1} B^{T}\right)^{-1} B C^{-1} B^{T} A^{-1}\right) B \\
=I+C^{-1} B^{T}\left(\left(A-B C^{-1} B^{T}\right)^{-1}\right. \\
\left.\quad \times\left(A-B C^{-1} B^{T}\right) A^{-1}-A^{-1}\right) B=I .
\end{gathered}
$$

Lemma 6. $J^{-1}$ If we write

$$
J=\left(\begin{array}{cc}
A & B \\
B^{T} & C
\end{array}\right), \quad J^{-1}=\left(\begin{array}{cc}
A^{\prime} & B^{\prime} \\
B^{\prime T} & C^{\prime}
\end{array}\right),
$$

then $C^{\prime}=\left(C-B^{T} A^{-1} B\right)^{-1}$.

Proof. Given the form of $J^{-1}$, we have $B^{T} B^{\prime}+C C^{\prime}=$ $I_{(N-M) \times(N-M)}$ and $A B^{\prime}+B C^{\prime}=0$. From the latter equation, we get $B^{\prime}=-A^{-1} B C^{\prime}$. Substitute into the first equation to get $-B^{T} A^{-1} B C^{\prime}+C C^{\prime}=I_{(N-M) \times(N-M)}$. Since the dimensions of the matrices all match, we get the desired result.

Now we can give the proof of Theorem 5 .

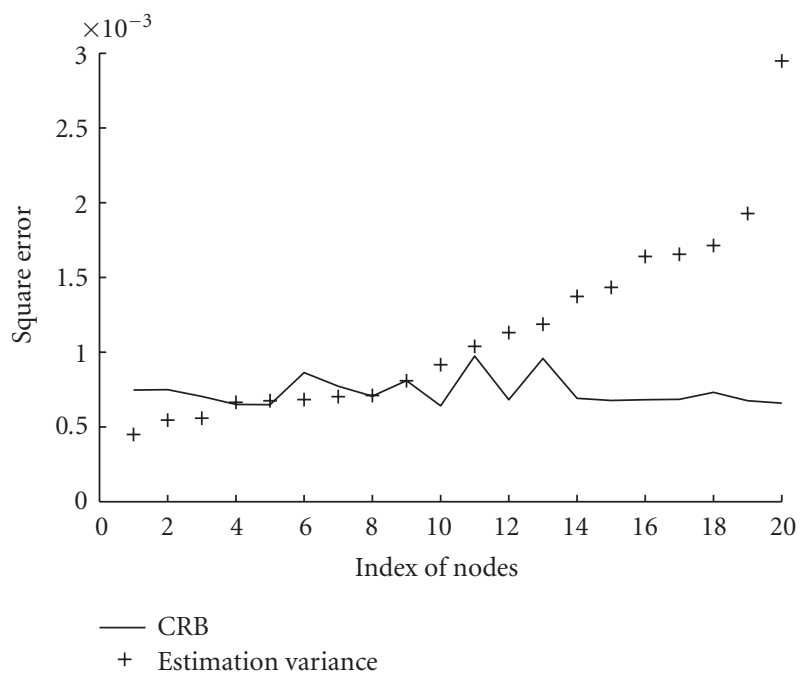

FIGURE 14: Comparison of CRB and estimation variance of a simple localization algorithm. Nodes are indexed by their estimation variance.

Proof. $C^{\prime}=\left(C-B^{T} A^{-1} B\right)^{-1}$, following Lemma 6 . Then from Lemma 5, we know that $\left(C-B^{T} A^{-1} B\right)^{-1}=C^{-1} B^{T}(A-$ $\left.B C^{-1} B^{T}\right)^{-1} B C^{-1}+C^{-1}$. Thus $C^{\prime}-C^{-1}=C^{-1} B^{T}(A-$ $\left.B C^{-1} B^{T}\right)^{-1} B C^{-1}=C^{-1}{ }^{T} B^{T}\left(A-B C^{-1} B^{T}\right)^{-1} B C^{-1}$. The second equality follows since $C^{T}=C$. Finally, $\left(A-B C^{-1} B^{T}\right)^{-1}$ is positive definite by Lemma 4 .

Definition 2 (upper-left submatrix). $1 \leq n \leq m$, the upperleft $n \times n$ submatrix of an $m \times m$ matrix $A$ is an $n \times n$ matrix $B$, s.t. $B(i, j)=A(i, j)$, for all $1 \leq i \leq n, 1 \leq j \leq n$.

Corollary 5 (monotonically increasing matrices). For a positive definite $N \times N$ matrix $J$, let $1 \leq n_{1} \leq n_{2} \leq \cdots \leq n_{M}=N$, let $A$ be the upper-left $n_{i} \times n_{i}$ submatrix of $A$, and let $B_{i}$ be the upper-left $n_{1} \times n_{1}$ submatrix of $A_{i}^{-1}$. Then

$$
A_{i}^{-1}=B_{1} \leq B_{2} \leq B_{3} \leq \cdots \leq B_{M}
$$

Proof. Notice that an upper-left submatrix of a positive definite matrix is still positive definite as shown in Lemma 3. Repeatedly applying Theorem 5 gives the desired result.

\section{B. A CASE STUDY OF A LOCALIZATION ALGORITHM}

The CRB only applies for unbiased estimators. To see why this is important, consider the simple localization scheme based on laceration and averaging that was proposed in [24]. To compare the CRB with the average estimation variance of our localization algorithm, we set up the sensor network as follows. All the sensors are located inside the unit circle. Three anchors are located at $(0,1),(\sqrt{3} / 2,-1 / 2)$, $(-\sqrt{3} / 2,-1 / 2)$. Twenty nodes with unknown positions are uniformly distributed inside the unit circle as shown in Figure 13.

Figure 14 compares the $\mathrm{CRB}$ on the estimation variance with the estimation variance for our simple localization 
scheme. $R_{\text {visible }}=2$ and the additive Gaussian errors have $\sigma=0.05$. The estimation variance for some nodes is smaller than the CRB for unbiased estimators because our localization scheme is biased.

\section{REFERENCES}

[1] C. Chang and A. Sahai, "Object tracking in a 2D UWB sensor network," in Proceedings of the 38th Conference on Signals, Systems and Computers (Asilomar '04), vol. 1, pp. 1252-1256, Pacific Grove, Calif, USA, November 2004.

[2] K. Seada, A. Helmy, and R. Govindan, "On the effect of localization errors on geographic face routing in sensor networks," in Proceedings of 3rd International Symposium on Information Processing in Sensor Networks (IPSN '04), pp. 71-80, Berkeley, Calif, USA, April 2004.

[3] L. Doherty, K. S. J. Pister, and L. El Ghaoui, "Convex position estimation in wireless sensor networks," in Proceedings of IEEE 20th Annual Joint Conference of the IEEE Computer and Communications Societies (IEEE INFOCOM '01), vol. 3, pp. 16551663, Anchorage, Alaska, USA, April 2001.

[4] C. Savarese, J. Rabaey, and K. Langendoen, "Robust positioning algorithms for distributed ad-hoc wireless sensor networks," in Proceedings of the General Track Annual Technical Conference (USENIX '02), pp. 317-327, Monterey, Calif, USA, June 2002.

[5] N. Bulusu, J. Heidemann, and D. Estrin, "GPS-less low-cost outdoor localization for very small devices," IEEE Personal Communications, vol. 7, no. 5, pp. 28-34, 2000.

[6] D. Niculescu and B. Nath, "Ad hoc positioning system (APS)," in Proceedings of IEEE Global Telecommunications Conference (Globecom '01), vol. 5, pp. 2926-2931, San Antonio, Tex, USA, November 2001.

[7] R. Nagpal, H. E. Shrobe, and J. Bachrach, "Organizing a global coordinate system from local information on an Ad Hoc sensor network," in Proceedings of 2nd International Workshop Information Processing in Sensor Networks (IPSN '03), vol. 2634 of Lecture Notes in Computer Science, pp. 333-348, Palo Alto, Calif, USA, April 2003.

[8] K. K. Chintalapudi, A. Dhariwal, R. Govindan, and G. Sukhatme, "Ad-hoc localization using ranging and sectoring," in Proceedings of 23th Annual Joint Conference of the IEEE Computer and Communications Societies (INFOCOM '04), vol. 4, pp. 2662-2672, Hong Kong, March 2004.

[9] R. L. Moses and R. Patterson, "Self-calibration of sensor networks," in Unattended Ground Sensor Technologies and Applications IV, vol. 4743 of Proceedings of the SPIE, pp. 108-119, Orlando, Fla, USA, April 2002.

[10] R. L. Moses, D. Krishnamurthy, and R. Patterson, "A selflocalization method for wireless sensor networks," EURASIP Journal on Applied Signal Processing, no. 4, pp. 348-358, 2003.

[11] J. Hightower, R. Want, and G. Boriello, "SpotON: an indoor 3d location sensing technology based on RF signal strength," Tech. Rep. UW CSE 00-02-02, University of Washington, Seattle, Wash, USA, February 2000.

[12] L. Girod and D. Estrin, "Robust range estimation using acoustic and multimodal sensing," in Proceedings of IEEE/RSJ International Conference on Intelligent Robots and Systems (IROS '01), vol. 3, pp. 1312-1320, Maui, Hawaii, USA, November 2001.

[13] A. Savvides, C.-C. Han, and M. B. Strivastava, "Dynamic finegrained localization in Ad-Hoc networks of sensors," in Pro- ceedings of the 7th Annual International Conference on Mobile Computing and Networking (MOBICOM '01), pp. 166-179, Rome, Italy, July 2001.

[14] C. Savarese, J. M. Rabaey, and J. Beutel, "Location in distributed ad-hoc wireless sensor networks," in Proceedings of IEEE International Conference on Acoustics, Speech, and Signal Processing (ICASSP '01), vol. 4, pp. 2037-2040, Salt Lake City, Utah, USA, May 2001.

[15] S. Capkun, M. Hamdi, and J.-P. Hubaux, "GPS-free positioning in mobile ad-hoc networks," in Proceedings of the 34th Annual Hawaii International Conference on System Sciences (HICSS '01), pp. 3481-3490, Maui, Hawaii, USA, January 2001.

[16] X. Ji and H. Zha, "Sensor positioning in wireless ad-hoc sensor networks using multidimensional scaling," in Proceedings of 23th Annual Joint Conference of the IEEE Computer and Communications Societies (INFOCOM '04), vol. 4, pp. 2652-2661, Hong Kong, March 2004.

[17] S. T. Smith, "Intrinsic Cramér-Rao bounds and subspace estimation accuracy," in Proceedings of the IEEE Sensor Array and Multichannel Signal Processing Workshop (SAM '00), pp. 489493, Cambridge, Mass, USA, March 2000.

[18] N. Patwari, A. O. Hero III, M. Perkins, N. S. Correal, and R. J. O'Dea, "Relative location estimation in wireless sensor networks," IEEE Transactions on Signal Processing, vol. 51, no. 8, pp. 2137-2148, 2003.

[19] A. Savvides, W. Garber, S. Adlakha, R. L. Moses, and M. B. Strivastava, "On the error characteristics of multihop node localization in ad-hoc sensor networks," in Proceedings of 2nd International Workshop Information Processing in Sensor Networks (IPSN '03), vol. 2634 of Lecture Notes in Computer Science, pp. 317-332, Palo Alto, Calif, USA, April 2003.

[20] H. Wang, L. Yip, K. Yao, and D. Estrin, "Lower bounds of localization uncertainty in sensor networks," in Proceedings of IEEE International Conference on Acoustics, Speech, and Signal Processing (ICASSP '04), Montreal, Quebec, Canada, May 2004.

[21] P. Stoica and T. L. Marzetta, "Parameter estimation problems with singular information matrices," IEEE Transactions on Signal Processing, vol. 49, no. 1, pp. 87-90, 2001.

[22] A. Dersan and Y. Tanik, "Passive radar localization by time difference of arrival," in Proceedings of Military Communications Conference (MILCOM '02), vol. 2, pp. 1251-1257, Anaheim, Calif, USA, October 2002.

[23] V. S. Chernyak, Fundamentals of Multisite Radar Systems, Gordon and Breach Science, Australia, 1998.

[24] C. Chang, "Localization and object-tracking in an ultrawideband sensor network," M.S. thesis, EECS Department, University of California Berkeley, Berkeley, Calif, USA, 2004.

[25] K. Hoffman and R. A. Kunze, Linear Algebra, Prentice-Hall, Englewood Cliffs, NJ, USA, 1992.

Cheng Chang received a B.E. degree from Tsinghua University, Beijing, in 2000, and an M.S. degree from University of California, Berkeley, in 2004. He is currently a Ph.D. student in UC Berkeley. His research interests are signal processing, control theory, and information theory. He is a Student Member of IEEE.

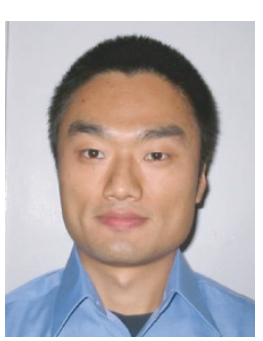


Anant Sahai received the B.S. degree in 1994 from UCB, the M.S. degree in 1996 from MIT, and the Ph.D. in 2001 from MIT. He joined the Department of Electrical Engineering and Computer Sciences at the University of California, Berkeley, in 2002 as an Assistant Professor. He is a Member of the Berkeley Wireless Research Center and the Wireless Foundations Center. In 2001, he spent a year as a research scientist


at the wireless startup Enuvis developing adaptive signal processing algorithms for extremely sensitive GPS receivers implemented using software-defined radio. Prior to that, he was a graduate student at the Laboratory for Information and Decision Systems at the Massachusetts Institute of Technology. His research interests are in wireless communication, signal processing, and information theory. He is particularly interested in fundamental questions of spectrum sharing among heterogeneous uses. 